\title{
Low-dimensional Dynamics of Two Coupled Biological Oscillators
}

\author{
Colas Droin\#, Eric R Paquet ${ }^{\#}$, Felix Naef ${ }^{\star}$ \\ Institute of Bioengineering, School of Life Sciences, Ecole Polytechnique Fédérale de Lausanne, \\ Lausanne $\mathrm{CH}-1015$, Switzerland \\ \# These authors contributed equally to this work.
}

\begin{abstract}
The circadian clock and the cell cycle are two biological oscillatory processes that coexist within individual cells. These two oscillators were found to interact, which can lead to their synchronization. Here, we develop a method to identify a low-dimensional stochastic model of the coupled system directly from time-lapse imaging in single cells. In particular, we infer the coupling and non-linear dynamics of the two oscillators from thousands of mouse and human single-cell fluorescence microscopy traces. This coupling predicts multiple phase-locked states showing different degrees of robustness against molecular fluctuations inherent to cellular-scale biological oscillators. For the 1:1 state, the predicted phase-shifts upon period perturbations were validated experimentally. Moreover, the phase-locked states are temperature-independent and evolutionarily conserved from mouse to human, hinting at a common underlying dynamical mechanism. Finally, we detect a signature of the coupled dynamics in a physiological context, explaining why tissues with different proliferation states exhibited shifted circadian clock phases.
\end{abstract}

\section{Keywords}

coupled oscillators; stochastic dynamics; phase-locking; synchronization; circadian clock; cell cycle

\begin{abstract}
The circadian clock and the cell cycle are two periodic processes that cohabit in many types of living cells. In single mammalian cells, circadian clocks consist of autonomous feedback loop oscillators ticking with an average period of about $24 \mathrm{~h}^{1}$, and controlling many downstream cellular processes ${ }^{2}$. In conditions of high proliferation such as those found in cultured cells or certain tissues, the cell cycle progresses essentially continuously and can
\end{abstract}

*Corresponding author: felix.naef@epfl.ch.

Data availability

The data supporting figures and other findings of this study are available from the corresponding author on request.

Code availability

The code is available online at the following URL:

https://github.com/ColasDroin/CouplingHMM.

Contributions

C.D., E.R.P., and F.N. designed and participated in the study concept. C.D. and E.R.P. developed computational analysis tools. E.R.P. performed the experiments. C.D. and E.R.P. processed and analyzed the experimental data. C.D., E.R.P., and F.N. interpreted the results. E.R.P. and F.N. acquired the funding. F.N. supervised the study. C.D., E.R.P., and F.N. wrote the manuscript. 
thus be abstracted as an oscillator with an average period matching the cell doubling time. Both processes fluctuate due to intra-cell molecular noise, as well as external fluctuations. While the precision of the circadian period is typically about $15 \%$ in fibroblast cells ${ }^{1}$, the cell cycle can be more variable depending on the conditions and cell lines ${ }^{3,4}$. Interestingly, previous work showed that the two cycles can mutually interact ${ }^{1}$, which may then lead, as theory predicts, to synchronized dynamics ${ }^{5,6}$ and important physiological consequences such as cell-cycle synchrony during liver regeneration ${ }^{7}$. In tissue-culture cells, which are amenable to systematic microscopy analysis, it was found that the phase dynamics of two oscillators shows phase-locking ${ }^{5,6}$, defined by a rational rotation number $p: q$ such that $p$ cycles of one oscillator are completed while the other completes $q$.

Concerning the nature of those interactions, the influence of the circadian clock on cell-cycle progression and division timing has been analyzed in several systems ${ }^{7-13}$. In contrast, we showed in mouse fibroblasts that the cell cycle strongly influences the circadian oscillator ${ }^{5}$, which was also investigated theoretically and linked with DNA replication in bacteria ${ }^{14}$. In addition, human cells can switch between a state of high cell proliferation with a damped circadian oscillator, to a state of low proliferation but robust circadian rhythms, depending on molecular interactions and activities of cell cycle and clock regulators ${ }^{15}$.

Here, we exploit the fact that the two coupled cycles evolve on a low dimensional and compact manifold (the flat torus) to fully characterize their dynamics. In particular, starting from a generic stochastic model for the interacting phases combined with fluorescence microscopy recordings from thousands of individual cells, we obtained a data-driven reconstruction of the coupling function describing how the cell cycle influences the circadian oscillator. This coupling phase-locks the two oscillators in a temperatureindependent manner, and only few of the deterministically predicted phase-locked states were stable against inherent fluctuations. Moreover, we established that the coupling between the two oscillators is conserved from mouse to human, and can override systemic synchronization signals such as temperature cycles. Finally, we showed in a physiological context how such coupling explains why mammalian tissues with different cell proliferation rates have shifted circadian phases.

\section{Modeling the dynamics of two coupled biological oscillators}

To study the phase dynamics of the circadian and cell cycle oscillators, we reconstructed a stochastic dynamic model of the two coupled oscillators from single-cell time-lapse microscopy traces of a fluorescent Rev-erba-YFP circadian reporter ${ }^{1,5}$. Our approach consists in explicitly modeling the measured fluorescent signals, using a set of stochastic ordinary differential equations (SODEs) whose parameters are estimated by maximizing the probability of observing the data over the entire set of cell traces (Methods). We here present the key components of the model (detailed in Supplementary Information).

\section{Phase model}

First, we represent the phase dynamics of the circadian oscillator ( $\theta=0$ corresponds to peaks of fluorescence) and cell cycle ( $\phi=0$ is the cytokinesis) $[0,2 \pi) \times[0,2 \pi)$ on a torus. Since we showed previously that the influence of the clock on the cell cycle was negligible 
in NIH3T3 cells ${ }^{5}$, we here model only how the cell-cycle progression influences the instantaneous circadian phase velocity $\omega_{\theta}$ using a general coupling function $F(\theta, \phi)$ (Fig. 1a). To account for circadian phase fluctuations and variability in circadian period length known to be present in single cells ${ }^{1,16}$, we added a phase diffusion term $\sigma_{\theta} d W_{t}$. For the cell-cycle phase, we assumed a piecewise linear and deterministic phase progression in between two successive divisions. The SODEs for the phases read:

$$
\left\{\begin{array}{l}
d \theta=\frac{2 \pi}{T_{\theta}} d t+F(\theta, \phi) d t+\sigma_{\theta} d W_{t} \\
d \phi=\frac{2 \pi}{T_{\phi}^{i}} d t
\end{array}\right.
$$

Here, $T_{\theta}$ represents the intrinsic circadian period, while the term $T_{\phi}^{i}$ represents the $i^{\text {th }}$ cellcycle interval between two successive divisions.

\section{Model of the signal}

We linked the circadian phase with the measured time traces through a $2 \pi$-periodic function $W(\theta)$. In addition, as suggested by typical data traces (Supplementary Fig. 1a), we considered amplitude $\left(A_{t}\right)$ and baseline $\left(B_{t}\right)$ fluctuations, which for simplicity we modeled as independent from $\theta_{t}$, an assumption that was supported a posteriori (Supplementary Information). The full model for the observed signal $S_{t}$ thus reads:

$$
S_{t}=e^{A_{t}} w\left(\theta_{t}\right)+B_{t}+\xi
$$

where $\xi$ is a normally distributed random variable (measurement noise) and $A_{t}, B_{t}$ are Ornstein-Uhlenbeck processes varying more slowly than the phase distortion caused by $F(\theta, \phi)$, i.e., on timescales on the order of the circadian period (Supplementary Information).

\section{Inference of phases \& coupling function}

From this stochastic model (Equations 1\&2, Fig. 1b), we built a Hidden Markov Model (HMM) to calculate posterior probabilities of the oscillator phases at each measured time point, using the forward-backward algorithm ${ }^{17}$. To estimate $F(\theta, \phi)$, we used a maximumlikelihood approach that combines goodness of fit with sparseness and smoothness constraints, which we implemented with an Expectation-Maximization (EM) algorithm (Methods, Supplementary Information).

The successive steps of our approach are illustrated in Figure 1c. Dividing cells indicated that, typically, the circadian phase progression shows variations in phase velocity (Supplementary Fig. 1a). To validate that these variations can identify $F(\theta, \phi)$, we generated noisy traces in silico with pre-defined $F(\theta, \phi)$ and reconstructed the coupling function, showing excellent qualitative agreement (Supplementary Fig. 1b-c).

\section{Influence of the cell cycle on the circadian phase}

In mouse embryonic fibroblasts (NIH3T3), we showed that due to the coupling, circadian periods decrease with temperature in dividing cells, but not in quiescent cells ${ }^{5}$. To further 
understand how temperature influences the interaction between the two oscillators, we reanalyzed NIH3T3 traces (24-72h long) obtained at $34^{\circ} \mathrm{C}, 37^{\circ} \mathrm{C}$, and $40^{\circ} \mathrm{C}^{5}$. From those, we found that both the inferred coupling functions and phase densities at the three temperatures were very similar, with almost identical 1:1 phase-locked orbits (Supplementary Fig. 2a-c). We therefore modeled the coupling as temperature-independent and re-constructed a definitive $F(\theta, \phi)$ from traces at all temperatures (Fig. 2a, Supplementary Fig. 2d). This function shows a diffuse structure mainly composed of two juxtaposed diagonal stripes: one for phase acceleration (red), and one, less structured, for deceleration (blue). The slopes of these stripes are about one, which indicates that an approximate minimal model of the coupling would be a function $F(\theta, \phi)=f(\theta-\phi)$. However, the phase velocity varies along the stripes and attractor (see below), which justifies using a 2D parameterization of the coupling function. The phase density for cells with fixed cell-cycle period of $22 \mathrm{~h}$ (corresponding to the mean cell-cycle period in the full dataset) (Fig. 2b, and Movie 1) clearly suggests $1: 1$ phase-locking. In fact, analyzing the predicted deterministic dynamics (Equation 1, with the reconstructed $F(\theta, \phi)$, and without the noise) shows a 1:1 attractor (Fig. 2c). Thus, in this $1: 1$ state, the endogenous circadian period of $24 \mathrm{~h}$ is shortened by two hours, which results from acceleration occurring after cytokinesis $(\phi=0)$ when the circadian phase is near $\theta \approx 0.8 \times 2 \pi$, and lasting for the entire G1 phase, until about $\theta \approx 0.4 \times 2 \pi$ when cells typically enter S phase $(\phi \approx 0.4 \times 2 \pi$ at the G1/S transition, Supplementary Information, Fig. 2d).

\section{Phase dynamics in perturbation experiments}

The reconstructed model allows us to simulate the circadian phase dynamics in function of the cell-cycle period, which is relevant as the cells display a significant range of cell-cycle lengths (Supplementary Fig. 3a). In the deterministic system, we find 1:1 phase-locking over a range of cell-cycle times varying from $19 \mathrm{~h}$ to $27 \mathrm{~h}$, showing that the cell cycle can both globally accelerate and slow down circadian phase progression (Fig. 3a). The attractor shifts progressively to the right in the phase-space, yielding a circadian phase at division ranging from $\theta \approx 0.7 \times 2 \pi$ at division when $T_{\phi}=19 h$ to $\theta \approx 0.9 \times 2 \pi$ when $T_{\phi}=27 h$. Since the attractor for different cell-cycle periods shifts, the circadian phase velocity profile also changes (Supplementary Fig. 3b). To validate the predicted shifts, we experimentally subjected cells to perturbations inducing a large variety of cell-cycle periods and compared the observed circadian phase to the model prediction at three different cell-cycle phases, revealing an excellent agreement, with no additional free parameters (Fig. 3b).

The simulations also clearly revealed multiple phase-locked states $(1: 2,1: 1,2: 1,3: 1$, etc, $p: q$ indicating the number of cell cycles $p$ and the number of clock cycles $q$ ), represented as Arnold tongues (Fig. 3c, and Movie 2 for an animated phase-space representation). We identified cell data trajectories following almost perfectly the deterministic attractors, both in the 1:1 and 1:2 phase-locking states (Fig. 3e and 3f, respectively); however, cells showing other $p: q$ states were rarely observed. 


\section{Fluctuations extend 1:1 phase-locking asymmetrically}

To understand the differences between the simulated deterministic system and observed cell traces, we simulated the stochastic dynamics (Equation 1). We then compared measured data trajectories stratified by cell-cycle periods (Fig. 4a) with deterministic (Fig. 4b) and stochastic simulations (Fig. 4c). This revealed that data agree better with stochastic than deterministic simulations, indicating that the phase fluctuations qualitatively change the phase portrait. One striking observation is the increased range of 1:1 phase-locking in the noisy system, however asymmetrically, since this occurs for shorter, but not for longer cellcycle periods. Indeed, while 1:2 phase-locking is observed in the data and the noisy simulations, the deterministically predicted 2:1 state is replaced in the data and stochastic system by 1:1-like orbits. Consistently, spectral analysis revealed significant differences between deterministic and stochastic simulations (Supplementary Fig. 4a-b, Movie 3); in addition, the coupling, specifically in the 1:1 state, was able to efficiently filter the noise (Supplementary Fig. 4b, right).

\section{Evolutionarily conserved phase-locking}

Most studies investigating the interaction between the cell cycle and the circadian oscillators in mammals are from rodent models ${ }^{1,5-8,18}$. To test if the above phase-locked dynamics are conserved in human U2OS cells, an established circadian oscillator model ${ }^{19,20}$, we engineered a U2OS cell line termed U2OS-Dual expressing a dual circadian fluorescent (Rev-erba-YFP) and luminescent (Bmal1-luc) reporter system. U2OS-Dual cells possess a functional circadian clock behaving similarly to NIH3T3 cells also expressing a Bmal1-Luc luminescent reporter ${ }^{21}$ (Fig. 5a). We scrutinize the relation between the two cell lines by comparing their behavior in different conditions: at $34^{\circ} \mathrm{C}$ and $37^{\circ} \mathrm{C}$ for cells with synchronized and non-synchronized circadian cycles ${ }^{5}$ (Fig. 5b-g).

Similarly to NIH3T3 cells, the division events of non-synchronized U2OS-Dual cells grown at $37^{\circ} \mathrm{C}$ occurred $4.96 \pm 2.6 \mathrm{~h}$ before a peak in the circadian fluorescent signal (Fig. $5 \mathrm{c}$ ), indicating that the cell cycle and the circadian clock interact. To investigate the directionality of this interaction, we tested, like in NIH3T3 cells ${ }^{5}$, whether the circadian clock phase could influence cell-cycle progression by resetting the circadian oscillator using dexamethasone (dex), a circadian resetting cue ${ }^{22}$ that does not perturb the cell cycle ${ }^{1}$. We found the expected resetting effect of dex on the circadian phase by the density of peaks in reporter levels during the first $10 \mathrm{~h}$ of recording, but with unnoticeable effects on the timing of the first division (Fig. 5d). However, the circadian peak following the first division occurred systematically around $5 \mathrm{~h}$ after the division in both conditions, suggesting that cell division in U2OS can reset circadian phases and overwrite dex synchronization. Synchronization of the circadian clocks for dex- $v s$ non- treated cells was expectedly higher for dex and gradually decreased to reach the level of the untreated cells (Fig. 5e), contrasting with the generally lower synchronization of cell divisions in both conditions. To then test if the cell cycle could influence the circadian clock, we lengthened the cell-cycle period by growing cells at $34^{\circ} \mathrm{C}$ and compared with $37^{\circ} \mathrm{C}$. Interestingly, cells at $34^{\circ} \mathrm{C}$ showed a longer circadian period compared to $37^{\circ} \mathrm{C}$ (Fig. $5 \mathrm{f}$ ), unlike the temperature compensated circadian periods $(\sim 25 \mathrm{~h})$ in non-dividing cells (Fig. 5a, g-h). 
Thus, similarly to mouse NIH3T3 cells, the coupling directionality is predominantly from the cell cycle to the circadian clock. In fact, the reconstructed coupling function for U2OSDual cells grown at $37^{\circ} \mathrm{C}$ (Fig. 5i) is structurally similar to that obtained in mouse fibroblasts (Fig. 2a), with the ensuing dynamics also showing a 1:1 attractor.

\section{Dividing cells lose circadian temperature entrainment}

In mammals, circadian clocks in tissues are synchronized by multiple systemic signals ${ }^{23}$. In fact, temperature oscillations mimicking those physiologically observed can phase-lock circadian oscillators in non-dividing (contact-inhibited) NIH3T3 cells in vitro ${ }^{24}$. To study how the found interactions influence temperature entrainment, we applied temperature cycles (24h period ranging from $35.5^{\circ} \mathrm{C}$ to $38.5^{\circ} \mathrm{C}$ ) to $\mathrm{U} 2 \mathrm{OS}$ cells growing at different rates (plated at different densities) and monitored population-wide Bmal1-luc signals (Fig. 6a). We found that, independently of initial densities, as the populations reach confluency, the phases and amplitudes become stationary, showing 1:1 entrainment (Fig. 6b, c and Supplementary Fig. 5a). During the initial transients, emerging circadian oscillations in nonconfluent cells showed phases that were already stationary, at least once cell numbers were sufficiently high to obtain reliable signals.

As cell confluence increases, the proportions of cells which stop cycling (exit to G0) increases $^{25}$. We therefore hypothesized that the observed phase and amplitude profiles in Bmal1-luc signals (Fig. 6b, c) originate from a mixture of two populations: an increasing population of non-dividing cells (G0) showing 'normal' entrainment properties, and dividing cells. We considered three scenarios for the dividing cells: i) the circadian oscillators in dividing cells adopt the same circadian profile as non-dividing entrained cells; ii) are not entrained; or iii) are entrained, but with a different phase compared to non-dividing cells (Supplementary Fig. 5b). These scenarios can be distinguished by the predicted phase and amplitude profiles (Supplementary Fig. 5c). Clearly, the measured profiles for U2OS-Dual cells favored the second scenario, suggesting that circadian oscillators in dividing cells do not entrain to the applied temperature cycles.

\section{Proliferation is associated with tissue-specific circadian phases}

The above findings suggest that phases or amplitudes of circadian clocks in organs in vivo might be influenced by the proliferation state of cells in the tissue. To test this, we investigated circadian clock parameters in different mouse tissues using a study of mRNA levels in twelve adult ( 6 weeks old males) mouse tissues, which revealed that clock phases span 1.5 hours between the earliest and latest tissues (Fig. 6d) ${ }^{26,27}$, an effect which is considered large in chronobiology as even period phenotypes of core clock genes are often smaller $^{2,28,29}$. We noticed that the mean mRNA levels across tissues of many genes correlated with the phase offsets (Supplementary Table 1). However, gene functions related to cell proliferation stood out as the most strongly enriched (Fig. 6f, Supplementary Table 1). Among those genes, the levels of known markers of cell proliferation such as Mki67 or Myc were strongly correlated with the phase offsets (Fig. 6g-h, Supplementary Table 1). Amplitudes, on the other hand, were not correlated with proliferation genes, but rather with neuronal specific genes, as expected owing to the damped rhythms present in those tissues 
(Fig. 6i, Supplementary Table 1) ${ }^{26}$. Thus, this analysis suggests that the differences in basal proliferation levels observed in normal tissues might underlie the dephasing of the circadian clock, suggesting a physiological role for the interaction of the cell cycle and circadian clocks.

\section{Discussion}

A goal in quantitative single-cell biology is to obtain data-driven and dynamical models of biological phenomena in low dimensions. In practice, the heterogeneity and complex physics underlying the emergence of biological function in non-equilibrium living systems, as well as the sparseness of available measurements pose challenges. Here, we studied a system of two coupled biological oscillators, sufficiently simple to allow data-driven model identification, yet complex enough to exhibit qualitatively distinct dynamics, i.e. $p: q$ states and quasi-periodicity.

In the coupled cell cycle and circadian oscillator system, phase-locked states different from $1: 1$ have been observed ${ }^{10}$. While multiple attractors, notably $1: 1$ and $3: 2$, were found in mouse NIH3T3 cells under transient dexamethasone stimulation ${ }^{6}$, we here report 1:2 states for long cell-cycle times under steady, unstimulated, conditions. Unlike other deterministically predicted states, 1:2 was sufficiently robust and observed in some cells. In fact, we found that noise extended the range of the 1:1 tongue, but asymmetrically, i.e. towards decreased cell-cycle periods. This may be reminiscent of generalized Poincaré oscillators showing that the entrainment range is broader for limit cycles with low relaxation rates ${ }^{30}$. Indeed, noise could decrease relaxation rates and thereby broaden Arnold tongues. In addition, for certain cell cycle periods, we observed the superposition of multiple states, both in the data and in the stochastic simulations, which were not present in the deterministic analysis (Fig. 4, see notably $\mathrm{T}=12 \mathrm{~h}$ and $\mathrm{T}=36 \mathrm{~h}$ ). This is reminiscent of mode hopping as described in the context of an oscillatory gene circuit underlying inflammatory responses ${ }^{31}$, however, here the corresponding Arnold tongues do not overlap in the range of the biologically relevant coupling strength $(K=1$, Fig. 3d).

While we focused on the emergent dynamics in the coupled oscillator system, considerations on possible biological mechanisms are relevant for follow-up biochemical analyses. How chromosome condensation or nuclear envelope breakdown may influence the circadian clock phase progression via either transcriptional shutdown, or displacement of chromatin bound circadian repressors, respectively, was discussed previously ${ }^{5}$. For example, Rev-erba transcription being so tightly locked to cell divisions (the peak accumulation of the reporter occurs $5 \mathrm{~h}$ after mitosis) could reflect the sudden derepression of its promoter, due to displaced CRYPTOCHROME1 (CRY1) containing repressor complex following nuclear envelope breakdown ${ }^{32}$. In turn, REV-ERB-A accumulation influences clock phase by binding to promoters of multiple core clock components, including $C_{r y} 1{ }^{33,34}$. More specific transcriptional activities could also play a key role in coupling the cell and circadian cycles. In fact, the circadian oscillator is exquisitely sensitive to numerous signaling pathways, impinging upon the clock by transcriptional induction of Period genes, which thus provides an efficient synchronization method ${ }^{22}$. Similarly, entrainment via temperature cycles also converges onto Period gene transcription ${ }^{24}$. However, we are not aware of cell-cycle 
dependent transcriptional regulators, such as E2F factors, targeting clock components like the Period genes. Finally, since the regulation of protein stability is important for clock function ${ }^{35}$, it is possible that phosphorylation-controlled proteolytic activities driving the cell cycle could target circadian phase regulators ${ }^{36}$, thereby mediating the observed coupling.

In mammals, the circadian oscillator in the suprachiasmatic nucleus $(\mathrm{SCN})$ is the pacemaker for the entire organism ${ }^{37}$, driving $24 \mathrm{~h}$ rhythms in activity, feeding, body temperature and hormone levels. In particular, the SCN can synchronize peripheral cell-autonomous circadian clocks located within organs across the body ${ }^{38}$. Consistent with theory ${ }^{39}$, in a physiological context of entrainment, the coupling of the cell cycle with the circadian clock can induce proliferation dependent phase-shifts, which we observed. Such phase shifts could reflect a homogenous behavior of all cells, or it could reflect heterogeneity of cell proliferation states, possibly leading to wave propagation. The phase shifts we observed in tissues were associated with low proliferation, i.e. non-pathological states of tissue homeostasis and cell renewal. For example, the liver or the adrenal gland showed a phase advance compared to fully quiescent tissues like the brain.

When cell proliferation is abnormally high such as in cancer, circadian clocks are often severely damped ${ }^{40}$. While this absence of a robust circadian rhythm in malignant tissue states may reflect non functional circadian oscillators due to mutations in clock genes ${ }^{41}$, the damped rhythms may also reflect circadian desynchrony of otherwise functional circadian oscillators. Such desynchrony would readily follow from the coupling between the cell-cycle and circadian oscillators we highlight here, in the presence of non-coherent cell-cycle progression.

Methodologically, the new approach to reconstruct a dynamical model for the coupled oscillator system has significant advantages over previous methods, notably strong assumptions such as the sparse and localized coupling are dispensable ${ }^{5}$. Compared with generic model identification techniques ${ }^{42}$, our approach models the raw data and its noise structure explicitly. In the future, such data-driven identification of dynamical models might reveal dynamical instabilities underlying ordered states in spatially extended systems, as occurring, for instance, during somitogenesis ${ }^{43}$.

\section{Methods}

\section{Cell lines}

All cell lines (U2OS-Dual, NIH3T3-Bmal1-Luc, and U2OS-PGK-Luc) were maintained in a humidified incubator at $37^{\circ} \mathrm{C}$ with 5\% $\mathrm{CO} 2$ using DMEM cell culture media supplemented with $10 \%$ fetal bovine serum (FBS) and 1\% penicillin-streptomycin-glutamine (PSG). One day before luminescence or fluorescence acquisitions, we replaced DMEM with FluoroBrite DMEM media supplemented with 10\% FBS and 1\% PSG. NIH3T3 perturbation experiments were generated in Bieler et $\mathrm{al}^{5}$. Briefly, they correspond to temperature changes (34, 37, and $40^{\circ} \mathrm{C}$ ), treatment with CDK1 (RO-3306, Sigma-Aldrich) and CDK1/2

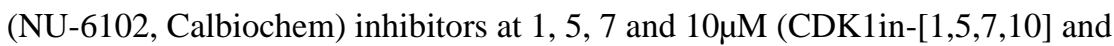
CDK2in-[1,5,7,10]), and shRNA-mediated knockdown of Cry2 (shCry). 


\section{Fluorescent time-lapse microscopy}

Time-lapse fluorescent microscopy for U2OS-Dual cells was performed at the biomolecular screening facility (BSF, EPFL) using an InCell Analyzer 2200 (GE healthcare). Experiments were performed at different temperature $\left(34^{\circ} \mathrm{C}, 37^{\circ} \mathrm{C}\right.$, or $\left.40^{\circ} \mathrm{C}\right)$ with a humidity and $\mathrm{CO}_{2}$ (5\%) control system. We used $100 \mathrm{~ms}$ excitation at 513/17 nm and emission at 548/22 $\mathrm{nm}$ to record the YFP channel. Cells were recorded by acquiring one field of view per well in a 96well black plate (GE healthcare). We used our previously developed semi-automated pipeline for segmentation and tracking of individual cells ${ }^{5}$. In total, traces from $n=551$ U2OS cells were obtained (typically 50 cells are obtained per movie). NIH3T3 single cells trace are reanalyzed from previous work ${ }^{5}$; here, we used $n=2504$ of those time traces. In all cases (NIH3T3 and U2OS), we followed several quality control metrics from ${ }^{5}$. Briefly, we discarded all traces that left the field-of-view at some point during the acquisition. We also visually inspected all traces, using a custom-made Matlab tool, to remove traces with problematic segmentation and tracking. In addition, we only kept traces with significant circadian amplitude (peak height $>0.25$, rescaled signals, Supplementary Information). To minimize boundary artifacts, typically, only traces with at least 2 full cell cycles were kept. The number of cells used for specific analyses, including sub-selections of traces based on the cell-cycle intervals, are indicate in the figure captions.

\section{Inferring the phase dynamics of two biological oscillators}

Denote by $\mathbf{D}$ the entire set of single cells traces comprising temporal intensity measurements ( $\Delta t=30 \mathrm{~min}$ ) from all fluorescent traces and $\Lambda$ the set of model parameters, comprising the gridded coupling function $F_{i j}$. Note that all parameters are shared by all cells in $\mathbf{D}$. To reconstruct the phase dynamics of our model, we seek to maximize the likelihood of the data $\mathscr{L}(\Lambda \mid \mathbf{D})$, that is, we solve:

$$
\Lambda^{*}=\operatorname{argmax} \mathscr{L}(\boldsymbol{\Lambda} \mid \mathbf{D})
$$

In practice, we used an EM algorithm, by iteratively optimizing the function $Q\left(\Lambda, \Lambda^{\prime}\right)$ over its first argument, where $Q$ can be written as follow:

$$
Q\left(\boldsymbol{\Lambda}, \boldsymbol{\Lambda}^{\prime}\right)=E\left[\log p(\mathbf{D}, \mathbf{X} \mid \boldsymbol{\Lambda}) \mid \mathbf{X}, \boldsymbol{\Lambda}^{\prime}\right]
$$

That is, $Q\left(\Lambda, \Lambda^{\prime}\right)$ corresponds to the expected value of the log-likelihood of the data with respect to the posterior probabilities of the hidden phases $\mathbf{X}$ (latent variables), computed using the current parameter $\Lambda^{\prime}$. This process guarantees a monotonous convergence of the log-likelihood, although a global maximum is not necessarily reached ${ }^{44}$.

To control for the many parameters $F_{i j}$, we added regularization constraints for both the smoothness and sparsity:

$$
Q_{p}\left(\boldsymbol{\Lambda}, \boldsymbol{\Lambda}^{\prime}\right)=Q\left(\boldsymbol{\Lambda}, \boldsymbol{\Lambda}^{\prime}\right)-\lambda_{1} \sum_{i j}\left\|F_{i j}\right\|^{2}-\lambda_{2} \sum_{i j} F_{i j}^{2}
$$


This expression is also guaranteed to converge $\mathrm{e}^{45}$.

Details about the optimization method, choice of the regularization parameters, and computation of the phase posteriors using a HMM are provided in Supplementary Information.

\section{Long-term temperature entrainment and luminescence recording}

We performed long-term temperature entrainment experiments using a Tecan plate reader Infinite $\mathrm{F} 200$ pro with a $\mathrm{CO}_{2}$ and temperature modules. One day before starting the experiment, serial dilution ranging from 40,000 to 2,500 cells were seeded in 96-well white flat bottom plates (Costar 3917). To prevent media evaporation, all wells were filled with $300 \mu \mathrm{l}$ of media composed of FluoroBrite, 10\% FBS, 1\% PSG, and $100 \mathrm{nM}$ D-luciferin (NanoLight technology) and covered with a sealing tape (Costar 6524). We set up temperature entrainment using stepwise increase (or decrease) of $0.5^{\circ} \mathrm{C}$ every 2 hours to produce temperature oscillating profiles going from $35.5^{\circ} \mathrm{C}$ to $38.5^{\circ} \mathrm{C}$ and back to $35.5^{\circ} \mathrm{C}$ again over a period of 24 hours. Intensities from all wells were recorded every 10 minutes with an integration time of 5000 milliseconds. Since temperature impacts the enzymatic activity of the luciferase ${ }^{46}$, we corrected the signal for this systematic effect (Supplementary Information).

\section{Association between gene expression and phase in tissues}

We used the average gene expression obtained from a selected set of twelve adult (6 weeks old males) mouse tissues from the Zhang et al. dataset (GEO accession GSE54650) ${ }^{26}$. For this analysis, we estimated the Pearson's correlation between the averaged gene expression and the circadian tissue phases or amplitudes reported in $\operatorname{ref}^{27}$. We selected the top 200 genes positively or negatively associated with either the phases or the amplitudes for gene ontology analysis ${ }^{47}$ (Supplementary Table 1).

\section{Supplementary Material}

Refer to Web version on PubMed Central for supplementary material.

\section{Acknowledgments}

We thank Rosamaria Cannavo for engineering the U2OS-Dual cell line and Jonathan Bieler for initial analyses. Fabien Kuttler from the Swiss Federal Institute of Technology (EPFL) Biomolecular Screening Facility (EPFLBSF) and Luigi Bozzo and José Artacho from the EPFL Bioimaging and Optics Core Facility (EPFL-BIOP) for assistance with the imaging. Fluorescence-activated cell sorting was performed at the EPFL Flow Cytometry Core Facility (EPFL-FCCF). This work was supported by the Swiss National Science Foundation Grant 310030_173079 and the EPFL. E.R.P was supported by a Canadian Institute of Health Research (CIHR 358808) and a SystemsX.ch Transition Postdoc Fellowships (51FSP0163584).

\section{References}

1. Nagoshi E, et al. Circadian gene expression in individual fibroblasts: cell-autonomous and selfsustained oscillators pass time to daughter cells. Cell. 2004; 119:693-705. DOI: 10.1016/ j.cell.2004.11.015 [PubMed: 15550250] 
2. Mermet J, Yeung J, Naef F. Systems Chronobiology: Global Analysis of Gene Regulation in a 24Hour Periodic World. Cold Spring Harbor perspectives in biology. 2017; 9doi: 10.1101/ cshperspect.a028720

3. Hahn AT, Jones JT, Meyer T. Quantitative analysis of cell cycle phase durations and PC12 differentiation using fluorescent biosensors. Cell cycle. 2009; 8:1044-1052. DOI: 10.4161/ cc.8.7.8042 [PubMed: 19270522]

4. Spencer SL, et al. The proliferation-quiescence decision is controlled by a bifurcation in CDK2 activity at mitotic exit. Cell. 2013; 155:369-383. DOI: 10.1016/j.cell.2013.08.062 [PubMed: 24075009]

5. Bieler J, et al. Robust synchronization of coupled circadian and cell cycle oscillators in single mammalian cells. Molecular systems biology. 2014; 10:739.doi: 10.15252/msb.20145218 [PubMed: 25028488]

6. Feillet $\mathrm{C}$, et al. Phase locking and multiple oscillating attractors for the coupled mammalian clock and cell cycle. Proceedings of the National Academy of Sciences of the United States of America. 2014; 111:9828-9833. DOI: 10.1073/pnas.1320474111 [PubMed: 24958884]

7. Matsuo T, et al. Control mechanism of the circadian clock for timing of cell division in vivo. Science. 2003; 302:255-259. DOI: 10.1126/science.1086271 [PubMed: 12934012]

8. Kowalska E, et al. NONO couples the circadian clock to the cell cycle. Proceedings of the National Academy of Sciences of the United States of America. 2013; 110:1592-1599. DOI: 10.1073/ pnas.1213317110 [PubMed: 23267082]

9. Mori T, Binder B, Johnson CH. Circadian gating of cell division in cyanobacteria growing with average doubling times of less than 24 hours. Proceedings of the National Academy of Sciences of the United States of America. 1996; 93:10183-10188. [PubMed: 8816773]

10. Yang Q, Pando BF, Dong G, Golden SS, van Oudenaarden A. Circadian gating of the cell cycle revealed in single cyanobacterial cells. Science. 2010; 327:1522-1526. DOI: 10.1126/ science.1181759 [PubMed: 20299597]

11. Matsu-Ura T, et al. Intercellular Coupling of the Cell Cycle and Circadian Clock in Adult Stem Cell Culture. Molecular cell. 2016; 64:900-912. DOI: 10.1016/j.molcel.2016.10.015 [PubMed: 27867006]

12. Plikus MV, et al. Local circadian clock gates cell cycle progression of transient amplifying cells during regenerative hair cycling. Proceedings of the National Academy of Sciences of the United States of America. 2013; 110:E2106-2115. DOI: 10.1073/pnas.1215935110 [PubMed: 23690597]

13. Gerard C, Goldbeter A. Entrainment of the mammalian cell cycle by the circadian clock: modeling two coupled cellular rhythms. PLoS computational biology. 2012; 8:e1002516.doi: 10.1371/ journal.pcbi.1002516 [PubMed: 22693436]

14. Paijmans J, Bosman M, Ten Wolde PR, Lubensky DK. Discrete gene replication events drive coupling between the cell cycle and circadian clocks. Proceedings of the National Academy of Sciences of the United States of America. 2016; 113:4063-4068. DOI: 10.1073/pnas.1507291113 [PubMed: 27035936]

15. Shostak A, et al. MYC/MIZ1-dependent gene repression inversely coordinates the circadian clock with cell cycle and proliferation. Nature communications. 2016; 7doi: 10.1038/ncomms 11807

16. Rougemont J, Naef F. Collective synchronization in populations of globally coupled phase oscillators with drifting frequencies. Physical review E, Statistical, nonlinear, and soft matter physics. 2006; 73doi: 10.1103/PhysRevE.73.011104

17. Rabiner LR, Juang B-H. An introduction to hidden Markov models. ieee assp magazine. 1986; 3:4-16.

18. Yeom M, Pendergast JS, Ohmiya Y, Yamazaki S. Circadian-independent cell mitosis in immortalized fibroblasts. Proceedings of the National Academy of Sciences of the United States of America. 2010; 107:9665-9670. DOI: 10.1073/pnas.0914078107 [PubMed: 20457900]

19. Vollmers C, Panda S, DiTacchio L. A high-throughput assay for siRNA-based circadian screens in human U2OS cells. PloS one. 2008; 3:e3457.doi: 10.1371/journal.pone.0003457 [PubMed: 18941634] 
20. Maier B, et al. A large-scale functional RNAi screen reveals a role for CK2 in the mammalian circadian clock. Genes \& development. 2009; 23:708-718. DOI: 10.1101/gad.512209 [PubMed: 19299560]

21. Nicolas D, Zoller B, Suter DM, Naef F. Modulation of transcriptional burst frequency by histone acetylation. Proceedings of the National Academy of Sciences of the United States of America. 2018; 115:7153-7158. DOI: 10.1073/pnas.1722330115 [PubMed: 29915087]

22. Balsalobre A, et al. Resetting of circadian time in peripheral tissues by glucocorticoid signaling. Science. 2000; 289:2344-2347. [PubMed: 11009419]

23. Dibner C, Schibler U, Albrecht U. The mammalian circadian timing system: organization and coordination of central and peripheral clocks. Annual review of physiology. 2010; 72:517-549. DOI: 10.1146/annurev-physiol-021909-135821

24. Saini C, Morf J, Stratmann M, Gos P, Schibler U. Simulated body temperature rhythms reveal the phase-shifting behavior and plasticity of mammalian circadian oscillators. Genes \& development. 2012; 26:567-580. DOI: 10.1101/gad.183251.111 [PubMed: 22379191]

25. Hayes $\mathrm{O}$, et al. Cell confluency is as efficient as serum starvation for inducing arrest in the G0/G1 phase of the cell cycle in granulosa and fibroblast cells of cattle. Animal reproduction science. 2005; 87:181-192. DOI: 10.1016/j.anireprosci.2004.11.011 [PubMed: 15911169]

26. Zhang R, Lahens NF, Ballance HI, Hughes ME, Hogenesch JB. A circadian gene expression atlas in mammals: implications for biology and medicine. Proceedings of the National Academy of Sciences of the United States of America. 2014; 111:16219-16224. DOI: 10.1073/ pnas.1408886111 [PubMed: 25349387]

27. Yeung J, et al. Transcription factor activity rhythms and tissue-specific chromatin interactions explain circadian gene expression across organs. Genome research. 2018; 28:182-191. DOI: 10.1101/gr.222430.117 [PubMed: 29254942]

28. Cermakian N, Monaco L, Pando MP, Dierich A, Sassone-Corsi P. Altered behavioral rhythms and clock gene expression in mice with a targeted mutation in the Period1 gene. The EMBO journal. 2001; 20:3967-3974. DOI: 10.1093/emboj/20.15.3967 [PubMed: 11483500]

29. Debruyne JP, et al. A clock shock: mouse CLOCK is not required for circadian oscillator function. Neuron. 2006; 50:465-477. DOI: 10.1016/j.neuron.2006.03.041 [PubMed: 16675400]

30. Granada AE, Herzel H. How to achieve fast entrainment? The timescale to synchronization. PloS one. 2009; 4:e7057.doi: 10.1371/journal.pone.0007057 [PubMed: 19774087]

31. Heltberg M, Kellogg RA, Krishna S, Tay S, Jensen MH. Noise Induces Hopping between NFkappaB Entrainment Modes. Cell systems. 2016; 3:532-539 e533. DOI: 10.1016/ j.cels.2016.11.014 [PubMed: 28009264]

32. Aryal RP, et al. Macromolecular Assemblies of the Mammalian Circadian Clock. Molecular cell. 2017; 67:770-782 e776. DOI: 10.1016/j.molcel.2017.07.017 [PubMed: 28886335]

33. Ukai-Tadenuma $\mathrm{M}$, et al. Delay in feedback repression by cryptochrome 1 is required for circadian clock function. Cell. 2011; 144:268-281. DOI: 10.1016/j.cell.2010.12.019 [PubMed: 21236481]

34. Mermet J, et al. Clock-dependent chromatin topology modulates circadian transcription and behavior. Genes \& development. 2018; 32:347-358. DOI: 10.1101/gad.312397.118 [PubMed: 29572261]

35. Siepka SM, et al. Circadian mutant Overtime reveals F-box protein FBXL3 regulation of cryptochrome and period gene expression. Cell. 2007; 129:1011-1023. DOI: 10.1016/ j.cell.2007.04.030 [PubMed: 17462724]

36. King RW, Deshaies RJ, Peters JM, Kirschner MW. How proteolysis drives the cell cycle. Science. 1996; 274:1652-1659. [PubMed: 8939846]

37. Hastings MH, Maywood ES, Brancaccio M. Generation of circadian rhythms in the suprachiasmatic nucleus. Nature reviews Neuroscience. 2018; 19:453-469. DOI: 10.1038/ s41583-018-0026-z [PubMed: 29934559]

38. Mohawk JA, Green CB, Takahashi JS. Central and peripheral circadian clocks in mammals. Annual review of neuroscience. 2012; 35:445-462. DOI: 10.1146/annurev-neuro-060909-153128

39. Pikovsky, ARMKJ. Synchronization. Cambridge University Press; 2001.

40. Shilts J, Chen G, Hughey JJ. Evidence for widespread dysregulation of circadian clock progression in human cancer. PeerJ. 2018; 6:e4327.doi: 10.7717/peerj.4327 [PubMed: 29404219] 
41. Kelleher FC, Rao A, Maguire A. Circadian molecular clocks and cancer. Cancer letters. 2014; 342:9-18. DOI: 10.1016/j.canlet.2013.09.040 [PubMed: 24099911]

42. Brunton SL, Proctor JL, Kutz JN. Discovering governing equations from data by sparse identification of nonlinear dynamical systems. Proceedings of the National Academy of Sciences of the United States of America. 2016; 113:3932-3937. DOI: 10.1073/pnas.1517384113 [PubMed: 27035946]

43. Soroldoni D, et al. Genetic oscillations. A Doppler effect in embryonic pattern formation. Science. 2014; 345:222-225. DOI: 10.1126/science.1253089 [PubMed: 25013078]

44. Dempster AP, Laird NM, Rubin DB. Maximum likelihood from incomplete data via the EM algorithm. Journal of the royal statistical society Series B (methodological). 1977:1-38.

45. Green PJ. On use of the EM for penalized likelihood estimation. Journal of the Royal Statistical Society Series B (Methodological). 1990:443-452.

46. Koksharov MI, Ugarova NN. Approaches to engineer stability of beetle luciferases. Computational and structural biotechnology journal. 2012; 2:e201209004.doi: 10.5936/csbj.201209004 [PubMed: 24688645]

47. Huang da W, Sherman BT, Lempicki RA. Bioinformatics enrichment tools: paths toward the comprehensive functional analysis of large gene lists. Nucleic acids research. 2009; 37:1-13. DOI: 10.1093/nar/gkn923 [PubMed: 19033363] 

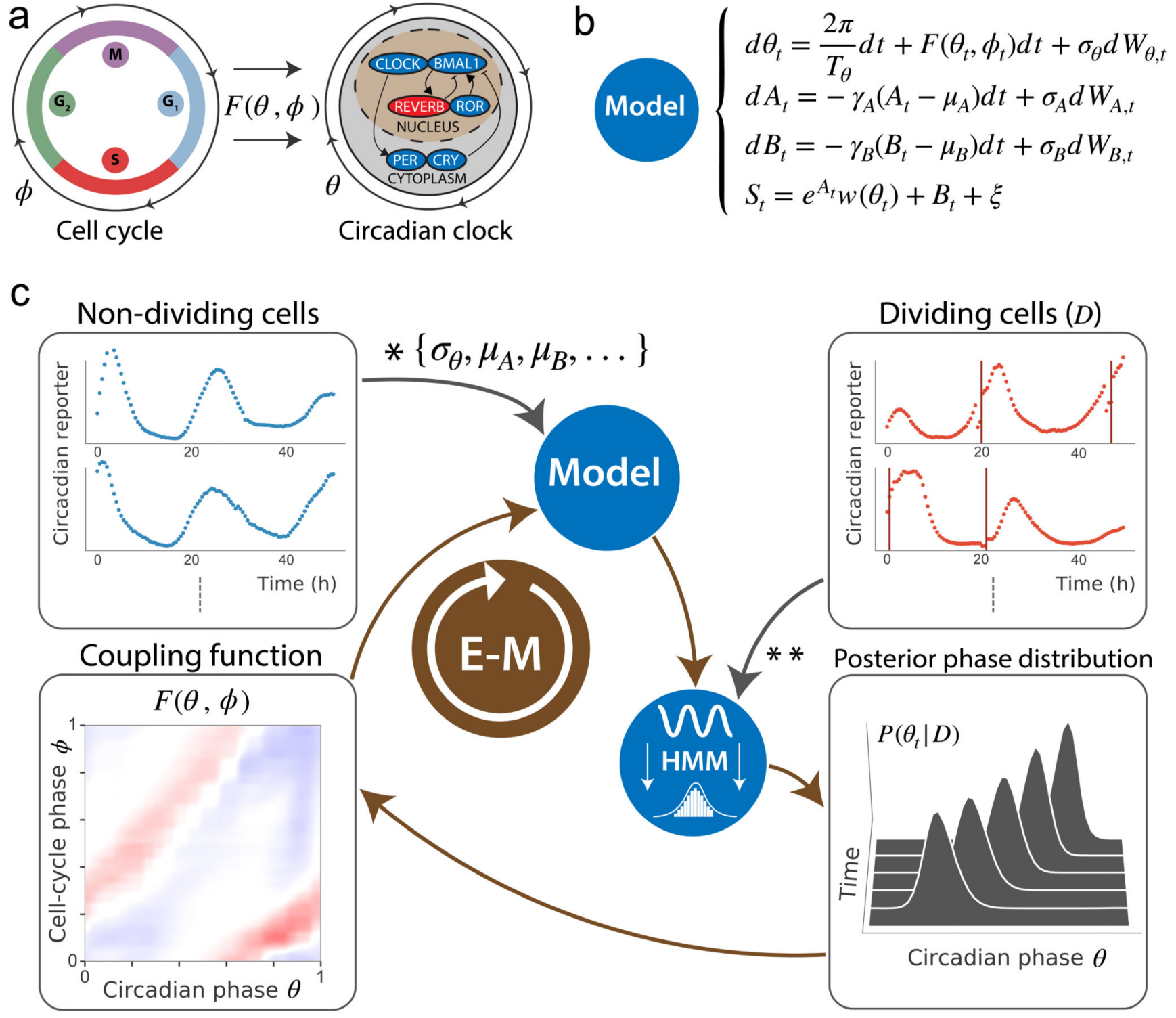

Figure 1. Reconstructing the phase dynamics and coupling of two biological oscillators.

(a) In mouse fibroblasts, the cell cycle (left) can influence the circadian oscillator (right) according to a coupling function $F(\theta, \phi)$, where $\phi$ denotes the cell cycle and $\theta$ the circadian oscillator phases. (b) Stochastic model for the signal $S_{t}$ using diffusion-drift SODEs for the circadian phase $\theta_{t}$, amplitude $A_{t}$ and background $B_{t}$ fluctuations, as well as a function $W(\theta)$ linking the phase $\theta_{t}$ to the measured observations, and $F(\theta, \phi)$ (c) Fluorescence microscopy traces (Rev-erba-YFP circadian reporter) are recorded for non-dividing and dividing cells (top left and top right boxes). Coupling-independent parameters (*) are estimated from nondividing cells while dividing cells are necessary to infer $F(\theta, \phi)(* *)$. The optimization problem is solved by converting the model to a HMM in which $\theta_{t}, A_{t}$ and $B_{t}$ are latent variables. The HMM is used on traces to compute posterior probabilities of circadian phases (bottom right box), while the cell-cycle phase is retrieved using linear interpolation between 
successive divisions (top right box, vertical orange lines). An iterative EM algorithm then yields the converged $F(\theta, \phi)$ (bottom left box). 

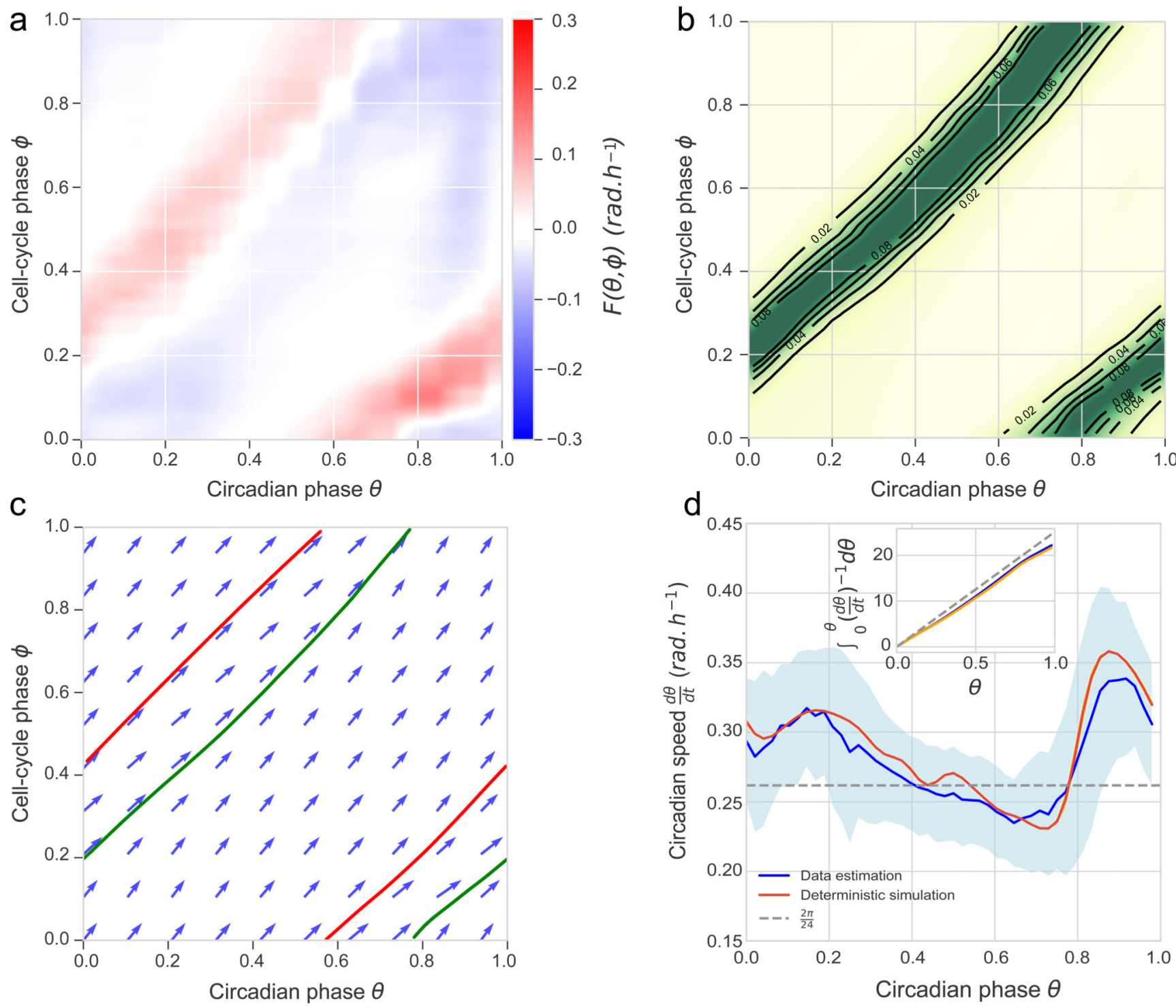

Figure 2. Influence of the cell cycle on the circadian phase enables 1:1 phase-locking.

(a) Coupling $F(\theta, \phi)$ optimized on dividing single-cell traces. Due to similar results

(Supplementary Fig. 2) traces from the three temperatures $\left(\mathrm{n}=154,271,302\right.$ traces at $34^{\circ} \mathrm{C}$, $37^{\circ} \mathrm{C}, 40^{\circ} \mathrm{C}$, resp.) are pooled. (b) Density of inferred phase traces from all the dividing traces with $22 \pm 1 \mathrm{~h}$ cell-cycle intervals indicates a 1:1 phase-locked state. (c) Numerical integration of phase velocity field (arrows, deterministic model) yields 1:1 attractor (green line) and repeller (red line). Here, the cell-cycle period was set to $22 \mathrm{~h}$. (d) Circadian phase velocity is not constant along the attractor, here for cells with $22 \pm 1 \mathrm{~h}$ cell-cycle intervals. Data (blue line, standard deviation in light-blue shading) and deterministic simulation (orange line). Inset: integrated time along the attractor. The gray line shows constant bare phase velocity $\omega_{\theta}=\frac{2 \pi}{24 h}$. 
a

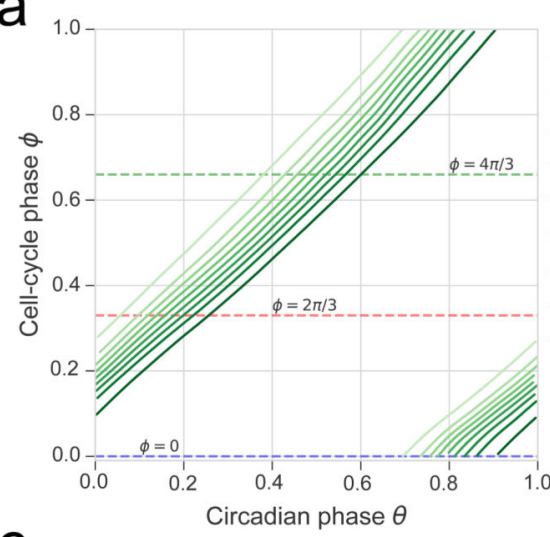

C

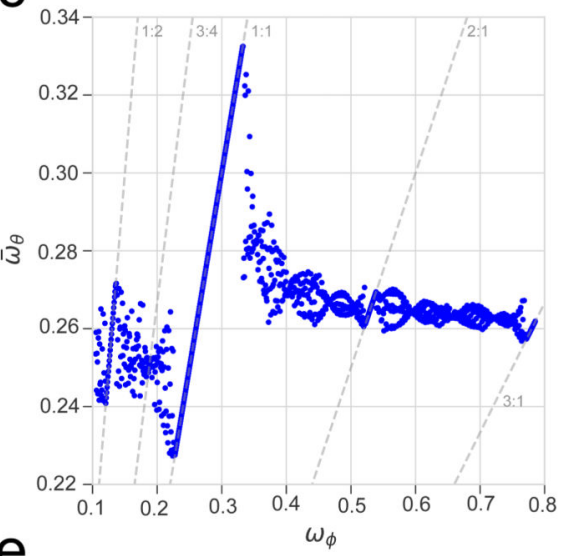

e

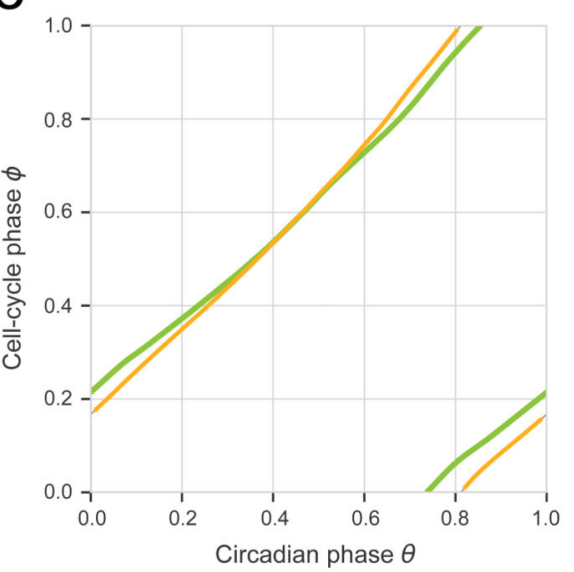

$\mathrm{b}$

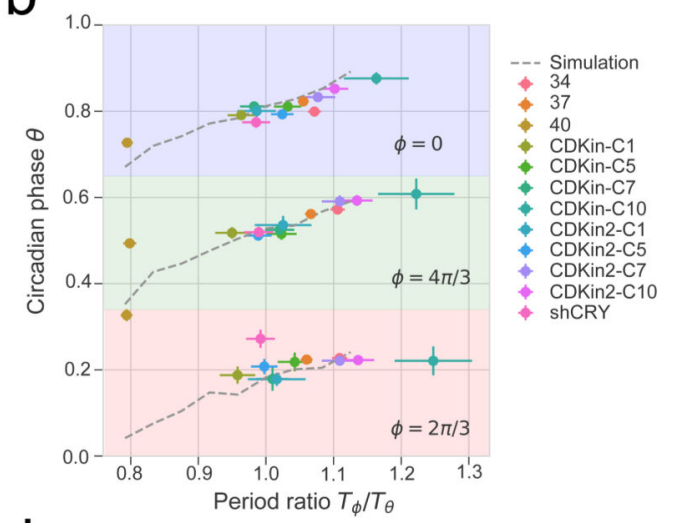

d

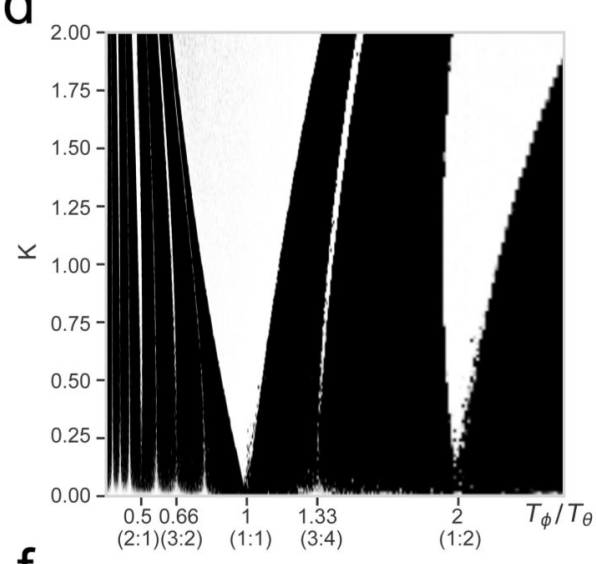

f

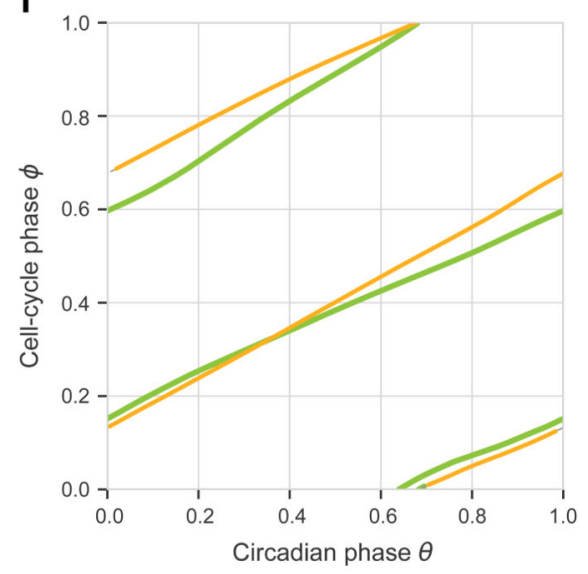

Figure 3. The coupling between the cell cycle and the circadian oscillator predicts phase shifts and phase-locking attractors in perturbation experiments.

(a) Simulated (deterministic) attractors for cell-cycle periods ranging from $19 \mathrm{~h}$ to $27 \mathrm{~h}$ show that the dephasing of the cell cycle and the circadian oscillator changes within the 1:1 state. Periods just outside of this range yield quasiperiodic orbits. The horizontal dashed lines indicate three different cell-cycle phases $\phi=0, \phi=\frac{1}{3} \times 2 \pi, \phi=\frac{2}{3} \times 2 \pi$ used in panel (b). (b)

Predictions from a) (dashed grey lines) against independent experimental data collected from 12 perturbation experiments (colored symbols, see legend, notation explained in 
Methods). (c) Multiple phased-locked states are predicted, recognizable by rational relationships between the frequencies of the entraining cell cycle and the entrained circadian oscillator, interspersed by quasiperiodic intervals. (d) Arnold tongues showing multiple phase-locked states in function of cell-cycle periods and coupling strength ( $K=1$ corresponds to the experimentally found coupling). Stable zones (white tongues) reveal attractors interspersed by quasi-periodicity. Although there are only two wider phased-locked state (1:1 and 1:2), several other $p: q$ states are found. (e-f) Representative single-cell traces (data in yellow) evolving near predicted attractors (green lines). A cell with $T_{\phi}=24 h$ (e) and one with $T_{\phi}=48 h(\mathbf{f})$ near the $1: 1$ and $1: 2$ orbits, respectively. 

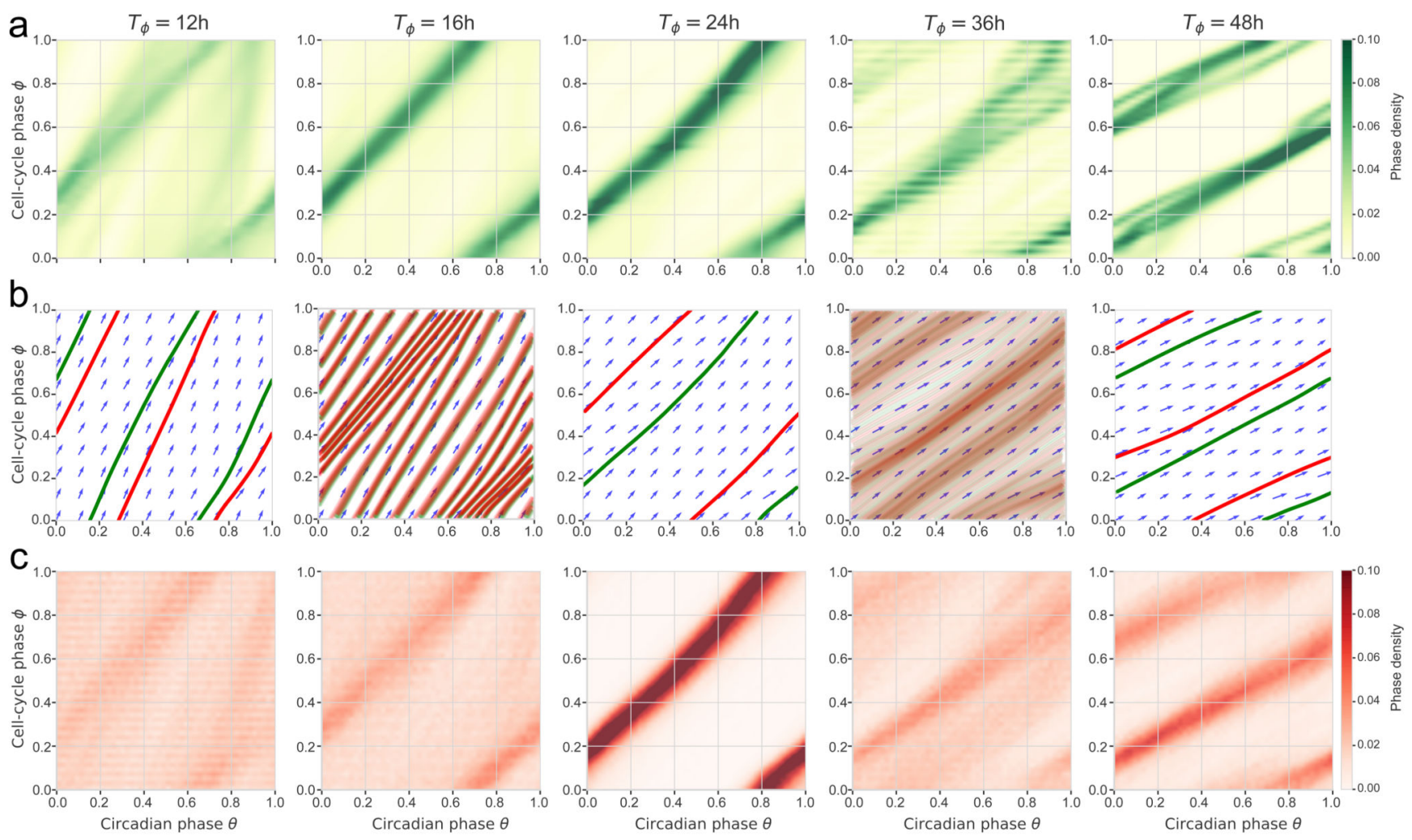

Figure 4. Single-cell data and stochastic simulations reveal robust 1:1 and 1:2 phase-locked states.

(a) Phase-space densities from the experimental traces stratified by cell-cycle periods $( \pm 1 \mathrm{~h}$ for each reference period); $\mathrm{n}=16,223,303,54,4$ cell traces in the $\mathrm{T}=12,16,24,36,48 \mathrm{~h}$ panels, resp. (b) Vector fields and simulated (deterministic) trajectories for the different cellcycle periods. Attractors are shown in green (forward time integration) and repellers (backward integration) in red (see also Movie 2). (c) Phase-space densities obtained from stochastic simulations of the model match better with the data compared to b). 

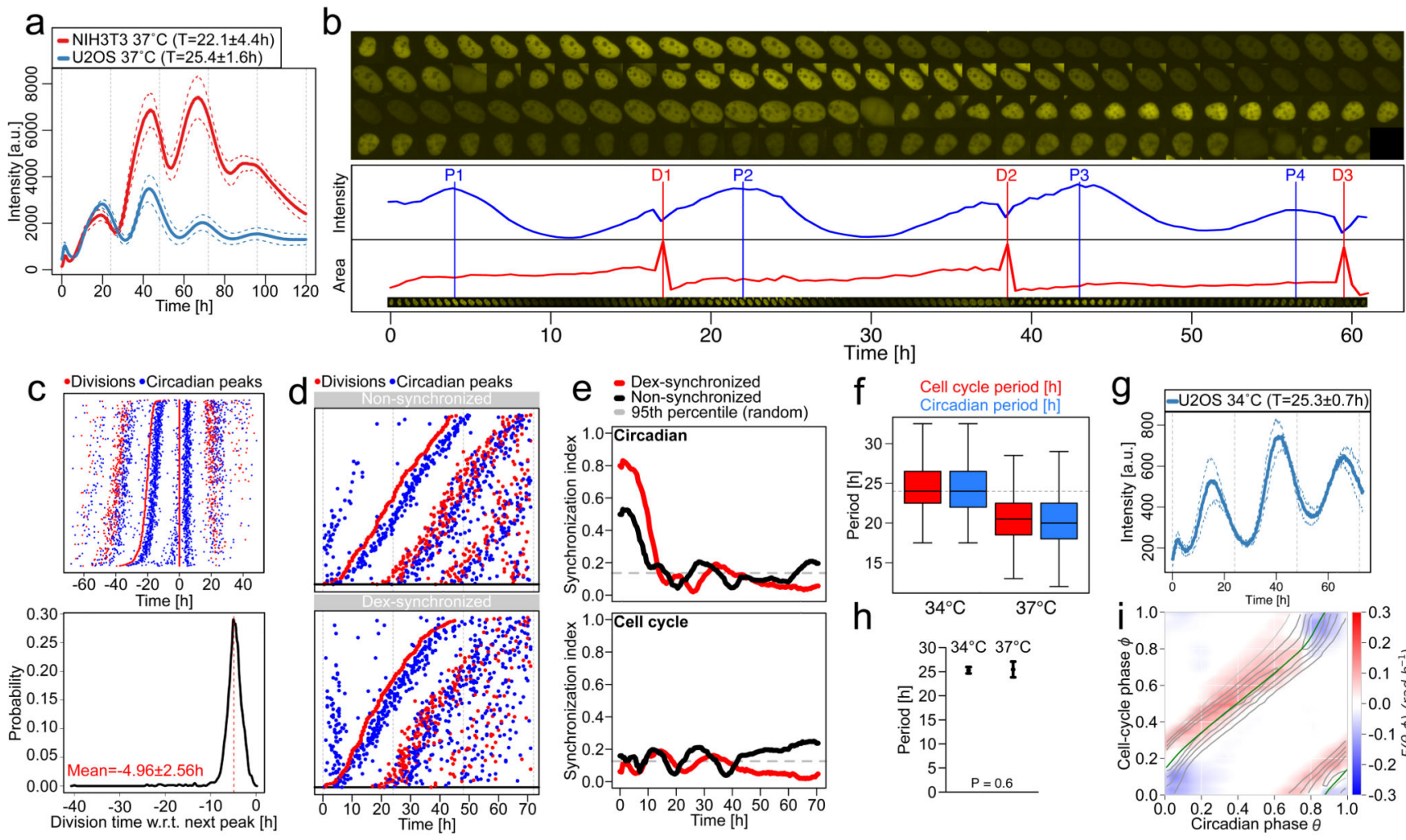

Figure 5. Conserved influence of the cell cycle on the circadian clock in human U2OS osteosarcoma cell.

(a) Mean luminescence intensities $( \pm \mathrm{SD}, \mathrm{n}=3)$ from non-dividing NIH3T3 and U2OS cells grown at $37^{\circ} \mathrm{C}$ expressing a Bmal1-Luc reporter. Values in the legend correspond to the mean periods \pm SD. (b) Semi-automated segmentation and tracking of U2OS cell lines expressing the Rev-erba-YFP circadian fluorescent reporter. Red vertical lines represent cell divisions (cytokinesis) and blue vertical lines show Rev-erba-YFP signal peaks. (c) Top: stack of divisions (red) and Rev-erba-YFP peaks (blue) for single U2OS traces centered on divisions. Bottom: distribution of the time of division relative to the next Rev-erba-YFP peaks (in read mean \pm SD, $n=1298$ ). (d) Divisions and Rev-erba-YFP peaks from single nonsynchronized (top), and dexamethasone (dex)-synchronized (bottom) U2OS traces ordered on the first division. (e) Synchronization index (SI) from non-synchronized (black) and dexsynchronized (red) traces for the circadian phase (top) and cell-cycle phase (bottom) estimated as in Bieler et al. ${ }^{5}$. The circadian SI from non-synchronized cells is relatively high due to plating. Dashed gray lines show $95^{\text {th }}$ percentiles of the SI for randomly shuffled traces. (f) Cell-cycle and circadian periods for U2OS cells grown at $34^{\circ} \mathrm{C}$ and $37^{\circ} \mathrm{C}(\mathrm{n}>90$ for all distributions). (g) Mean luminescence intensities ( $\pm \mathrm{SD}, \mathrm{n}=3$ ) for non-dividing U2OS cells grown at $34^{\circ} \mathrm{C}$ expressing a Bmal1-Luc reporter. Values in the legend correspond to the mean periods \pm SD. (h) Mean and standard deviation of the circadian period for non-dividing U2OS cells grown at $34^{\circ} \mathrm{C}$ and $37^{\circ} \mathrm{C}$ ( $\mathrm{n}=8$ at 34 and $\mathrm{n}=9$ at 37 , two-sided Wilcoxon's test). (i) Coupling function $F(\theta, \phi)$ optimized on $\mathrm{n}=551$ dividing $\mathrm{U} 2 \mathrm{OS}$ traces grown at $37^{\circ} \mathrm{C}$, superimposed with the attractor $\left(T_{\phi}=22 \mathrm{~h}\right.$ ) obtained from deterministic simulations (green line). 

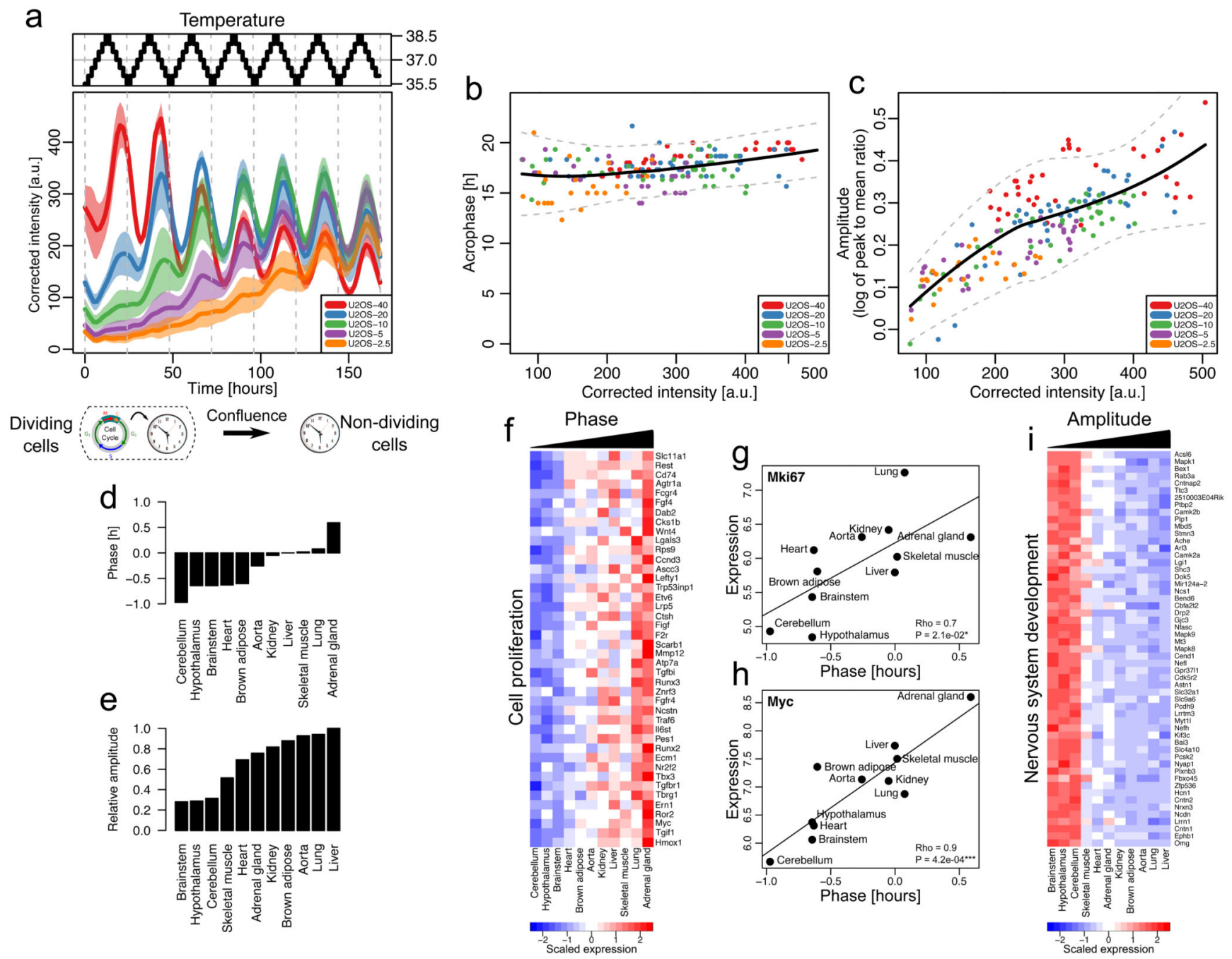

Figure 6. Temperature cycles do not entrain circadian oscillators in dividing cells and proliferation genes are associated with tissue-specific circadian phases.

(a) Corrected and averaged Bmal1-Luc intensities and 95\% confidence intervals $(n=6)$ from U2OS-Dual cells plated at different initial densities and subjected to a temperature entrainment (top). (b) Acrophases (times of the local peaks in luminescence) of the Bmal1Luc signal in function of the reporter intensity for the cells in a). Loess fit (black) and 95\% confidence intervals (gray). (c) Amplitude (log of peak to mean ratio) of the Bmal1-Luc oscillations in function of the reporter intensity for the cells in a). Loess fit (black) and $95 \%$ confidence intervals (gray). (d-e) Circadian phases (d) and amplitudes (e) of different mouse tissues obtained in ref $^{27}$, relative to liver. (f) Expression levels of genes positively associated with phases from d) and linked to cell proliferation. (g-h) Correlations between Mki67(g) and Myc (h) mRNA expression and circadian phases across mouse tissues (Pearson's correlation, two-sided P-values from t-distribution with n-2 degrees of freedom). (i) Expression levels of genes negatively associated with amplitudes and linked to nervous system development. 